\title{
Parameter Estimation of Nonlinear Large Scale Systems through Stochastic Methods and Measurement of its Dynamic Response
}

\author{
Dimitrios Giagopoulos ${ }^{1}$, Alexandros Arailopoulos ${ }^{1}$ \\ ${ }^{1}$ Department of Mechanical Engineering, University of Western Macedonia \\ Kozani 50100, Greece \\ e-mail: dgiagopoulos@uowm.gr, aarailopoulos@uowm.gr
}

Keywords: Large Scale Models, Nonlinear Dynamics, System Identification, Substructuring.

\begin{abstract}
In this work, a computational framework is presented in order to estimate the optimal values of the linear and nonlinear components of large scale dynamical systems using vibration measurements. An extensible framework for Bayesian Uncertainty Quantification and Propagation of complex and computationally demanding physical models, was connected in an efficient way with a numerical code leading to an automated determination of dynamic response of linear and nonlinear mechanical systems. The effect of correlation in the prediction error models postulated in the Bayesian model selection and parameter estimation technique is investigated. The measurements are taken to be either response time histories or frequency response functions. The framework was applied in two complex mechanical systems. First examined a lightweight bicycle frame with the nonlinear front suspension component. In this system uncertainty models of the linear and nonlinear suspension components are identified using the experimentally obtained transmissibility functions. These uncertainties, integrated with uncertainties in the linear frame of the bicycle, are propagated in order to estimate the uncertainties for the combined system. The second system examined include a two stage gear transmission system. In this model the housing of the gearbox is modeled by using finite elements, while the essential effects of the gear-pair, the bearings and the shafts are taken into account via a lumped nonlinear mathematical model. This model possesses strongly nonlinear characteristics, accounting for gear backlash, meshing stiffness, transmission error properties and bearing stiffness nonlinearities. In order to identify the values of the parameters, accelerations time histories are used, obtained during various operating conditions of the gearbox. These measurements are recorded from a special experimental device (Drivetrain Prognostics Simulator). The computational challenges are outlined and the effectiveness of the framework on these two complex mechanical systems is demonstrated.
\end{abstract}




\section{INTRODUCTION}

The main objective of the present work is to demonstrate the advantages of applying appropriate numerical and experimental methodologies in order to identify, update and optimize the model parameters and develop a high fidelity finite element model of both linear and non-linear components, comprising the whole assembly of the examined structure. In this process, many issues are taken into account, related to the development of FE model, the experimental modal analysis procedure and the development of effective computational model updating techniques.

The equations of motion of mechanical systems with complex geometry are first set up, applying classical finite element techniques. As the order of these models increases, the existing numerical and experimental methodologies for a systematic determination of their dynamic response become inefficient to apply. Therefore, there is a need for the development, improvement and application of new suitable methodologies for investigating dynamics of large-scale mechanical models in a systematic and efficient way. Moreover, in order to optimize the FE model of a structure, structural model updating methods [11], have been proposed in order to reconcile the numerical (FE) model, with experimental data. Structural model parameter estimation based on measured modal data (e.g. [4-10]) are often formulated as weighted least-squares estimation problems in which metrics, measuring the residuals between measured and model predicted modal characteristics, are build up into a single weighted residuals metric formed as a weighted average of the multiple individual metrics using weighting factors. Standard gradient-based optimization techniques are then used to find the optimal values of the structural parameters that minimize the single weighted residuals metric representing an overall measure of fit between measured and model predicted modal characteristics. Due to model error and measurement noise, the results of the optimization are affected by the values assumed for the weighting factors.

In this work, the applicability and effectiveness of the updating methods, coupled with robust, accurate and efficient finite element analysis software are applied on linear and nonlinear components of a whole structure using experimentally identified modal data. The framework was applied in two complex mechanical systems. First examined a lightweight bicycle frame with the nonlinear front suspension component. The second system examined include a two stage gear transmission system. In this work was selected to be presented in detail only the results of the first system. More specifically, a lightweight and geometrically complex bicycle frame as well as the suspension-fork subassembly of a real bicycle are examined, comprising the linear and nonlinear subsystems of the whole bike assembly. Furthermore, the suspension-fork subassembly is consisted of two linear parts (upper and lower fork part) connected with two springs and two seals which impose strong nonlinearity in the system. Issues related to estimating unidentifiable solutions [12-15] arising in FE model updating formulations are also addressed. A systematic study is carried out to demonstrate the effect of model error, finite element model parameterization, number of measured modes and number of mode shape components on the optimal models and their variability. It is demonstrated that the updated finite element models obtained using measured modal data may vary considerably.

The organization of this paper is as follows. First in the first section, the theoretical formulation of finite element model updating based on modal characteristics and frequency response functions is briefly presented. In the second section, the experimental device is introduced. First, a quick presentation of the digitization of the bicycle components leading to the final parametric CAD model is shown with the corresponding detailed FE models. Next presented the experimental modal analysis procedure followed in order to identify the modal 
characteristics and the FRF's. Finally, the parametric studies on updating the linear and nonlinear FE models of the bicycle components, with the predictions of frequency response functions and time histories, based on the optimal models, are presented in the fourth section. Conclusions are summarized in the fifth section.

\section{FINITE ELEMENT MODEL UPDATING METHODS}

\subsection{Modal Residuals}

Let $D=\left\{\hat{\omega}_{r}, \hat{\phi}_{r} \in R^{N_{o}}, r=1, \ldots, m\right\}$ be the measured modal data from a structure, consisting of modal frequencies $\hat{\omega}_{r}$ and mode shape components $\underline{\hat{\phi}}_{r}$ at $N_{o}$ measured DOFs, where $m$ is the number of observed modes. Consider a parameterized class of linear structural models used to model the dynamic behavior of the structure and let $\underline{\theta} \in R^{N_{\theta}}$ be the set of free structural model parameters to be identified using the measured modal data. The objective in a modal-based structural identification methodology is to estimate the values of the parameter set $\underline{\theta}$ so that the modal data $\left\{\omega_{r}(\underline{\theta}), \underline{\phi_{r}}(\underline{\theta}) \in R^{N_{0}}, r=1, \ldots, m\right\}$ predicted by the linear class of models at the corresponding $N_{0}$ measured DOFs best matches the experimentally obtained modal data in $D$. For this, let

$$
\varepsilon_{\omega_{r}}(\underline{\theta})=\frac{\omega_{r}^{2}(\underline{\theta})-\hat{\omega}_{r}^{2}}{\hat{\omega}_{r}^{2}} \text { and } \varepsilon_{\underline{\phi}_{r}}(\underline{\theta})=\frac{\left\|\beta_{r}(\underline{\theta}) \underline{\phi}_{r}(\underline{\theta})-\underline{\hat{\phi}}_{r}\right\|}{\left\|\hat{\phi}_{r}\right\|}
$$

be the measures of fit or residuals [16] between the measured modal data and the model predicted modal data for the $r$-th modal frequency and mode shape components, respectively, where $\|\underline{z}\|^{2}=\underline{z}^{\mathrm{T}} \underline{z}$ is the usual Euclidean norm, and $\beta_{r}(\underline{\theta})=\underline{\hat{\phi}}_{r}^{T} \underline{\phi}_{r}(\underline{\theta}) /\left\|\underline{\phi_{r}}(\underline{\theta})\right\|^{2}$ is a normalization constant that guaranties that the measured mode shape $\hat{\phi}_{r}$ at the measured DOFs is closest to the model mode shape $\beta_{r}(\underline{\theta}) \underline{\phi}_{r}(\underline{\theta})$ predicted by the particular value of $\underline{\theta}$. To proceed with the model updating formulation, the measured modal properties are grouped into two groups. The first group contains the modal frequencies while the second group includes the mode shape components for all modes. For each group, a norm is introduced to measure the residuals of the difference between the measured values of the modal properties involved in the group and the corresponding modal values predicted from the model class for a particular value of the parameter set $\underline{\theta}$. For the first group, the measure of fit $J_{1}(\underline{\theta})$ is selected to represent the difference between the measured and the model predicted frequencies for all modes. For the second group, the measure of fit $J_{2}(\underline{\theta})$ is selected to represent the difference between the measured and the model predicted mode shape components for all modes. Specifically, the two measures of fit are given by

$$
J_{1}(\underline{\theta})=\sum_{r=1}^{m} \varepsilon_{\omega_{r}}^{2}(\underline{\theta}) \text { and } J_{2}(\underline{\theta})=\sum_{r=1}^{m} \varepsilon_{\underline{\phi}_{r}}^{2}(\underline{\theta})=\sum_{r=1}^{m}\left[1-M A C_{r}^{2}(\underline{\theta})\right]
$$

where $\operatorname{MAC}_{r}(\underline{\theta})=\underline{\phi}_{r}^{T} \underline{\hat{\phi}}_{r}(\underline{\theta}) /\left\|\underline{\phi}_{r}\right\|\left\|\hat{\phi}_{r}\right\|$ is the Modal Assurance Criterion [23] between experimentally identified and estimated mode shapes for the $r$-th mode. Alternative measures of fit can easily be used and found in literature [18-22]. 


\subsection{Response Residuals}

Derived from the MAC for any measured frequency point, $\omega_{k}$ a global correlation coefficient may be used [17, 24]:

$$
x_{s}\left(\omega_{k}\right)=\frac{\left|\left\{H_{X}\left(\omega_{k}\right)\right\}^{H}\left\{H_{A}\left(\omega_{k}\right)\right\}\right|^{2}}{\left(\left\{H_{X}\left(\omega_{k}\right)\right\}^{H}\left\{H_{X}\left(\omega_{k}\right)\right\}\right)\left(\left\{H_{A}\left(\omega_{k}\right)\right\}^{H}\left\{H_{A}\left(\omega_{k}\right)\right\}\right)}
$$

where $\left\{H_{X}\left(\omega_{k}\right)\right\}$ and $\left\{H_{A}\left(\omega_{k}\right)\right\}$ are the experimental (measured) and the analytical (predicted) response vectors at matching excitation - response locations. As the MAC value, $x_{s}\left(\omega_{k}\right)$ assumes a value between zero and unity and indicates perfect correlation with $x_{s}\left(\omega_{k}\right)=1$. For $x_{s}\left(\omega_{k}\right)=0$, no correlation exists. Similar to the MAC, $x_{s}\left(\omega_{k}\right)$ is unable to detect scaling errors and is only sensitive to discrepancies in the overall deflection shape of the structure. To emphasis this characteristic, $x_{s}\left(\omega_{k}\right)$ is accordingly called the shape correlation coefficient [24].

The lack of sensitivity to scaling of the shape correlation coefficient does not allow the identification of identical FRFs. This insufficiency becomes even more dramatic if just one measurement and its corresponding prediction are correlated. In this case, the column vectors reduce to scalars and $\left\{H_{A}\left(\omega_{k}\right)\right\}=k\left\{H_{X}\left(\omega_{k}\right)\right\}$ is always satisfied (constant k may be complex), therefore leading to $x_{s}=1$ across the full frequency spectrum for uncorrelated FRFs.

As a result, a supplementary correlation coefficient $x_{a}\left(\omega_{k}\right)$ is proposed by targeting the discrepancies in amplitude. The amplitude correlation coefficient is defined as:

$$
x_{a}\left(\omega_{k}\right)=\frac{2\left|\left\{H_{X}\left(\omega_{k}\right)\right\}^{H}\left\{H_{A}\left(\omega_{k}\right)\right\}\right|}{\left(\left\{H_{X}\left(\omega_{k}\right)\right\}^{H}\left\{H_{X}\left(\omega_{k}\right)\right\}\right)+\left(\left\{H_{A}\left(\omega_{k}\right)\right\}^{H}\left\{H_{A}\left(\omega_{k}\right)\right\}\right)}
$$

where the response vectors are identical to those used for $x_{s}\left(\omega_{k}\right)$. As for the shape correlation coefficient, $x_{a}\left(\omega_{k}\right)$ is defined to lie between zero and unity. This time, however, the correlation measure is more stringent and only becomes unity if $\left\{H_{A}\left(\omega_{k}\right)\right\}=\left\{H_{X}\left(\omega_{k}\right)\right\}$. That is to say, all elements of the response vectors must be identical in both phase and amplitude even if only one measurement is considered. Similarly to modal residuals, two measures of fit are proposed using $x_{s}\left(\hat{\omega}_{r}\right)$ and $x_{a}\left(\hat{\omega}_{r}\right)$ which correspond to the identified resonant frequencies of the system:

$$
J_{3}(\underline{\theta})=\sum_{r=1}^{m}\left[1-x_{s}\left(\hat{\omega}_{r}, \underline{\theta}\right)^{2}\right] \text { and } J_{4}(\underline{\theta})=\sum_{r=1}^{m}\left[1-x_{a}\left(\hat{\omega}_{r}, \underline{\theta}\right)^{2}\right]
$$

\subsection{Weighting Residuals Method}

Minimizing at global minimum the following single objective, traditionally solves the parameter estimation problem:

$$
J(\underline{\theta} ; \underline{w})=w_{1} J_{1}(\underline{\theta})+w_{2} J_{2}(\underline{\theta})+w_{3} J_{3}(\underline{\theta})+w_{4} J_{4}(\underline{\theta})
$$

formed by the four objectives $J_{i}(\underline{\theta})$, using the weighting factors $w_{i} \geq 0, i=1,2,3,4$, with $w_{1}+w_{2}+w_{3}+w_{4}=1$. The objective function $J(\underline{\theta} ; \underline{w})$ represents an overall measure of fit between the measured and the model predicted characteristics. The relative importance of the residual errors in the selection of the optimal model is reflected in the choice of the weights. The 
results of the identification depend on the weight values used. The optimal solutions for the parameter set $\underline{\theta}$ for given $\underline{w}$ are denoted by $\underline{\hat{\theta}}(\underline{w})$ [12-15].

\section{EXPERIMENTAL APPLICATION}

The model updating methodologies are applied in order to update the FE model of a real bicycle. The bicycle consisted of the main frame, which presented in Figure 1 and is made of aluminum and from the front suspension which also consisted of two main parts which are presented respectively in Figures 3 and 4. The lower part of the fork, has the same material properties with the bicycle frame, while the upper fork part, consisted of steel tubes which are connected with a solid aluminum part. The thicknesses of the cross sections of the main frame as long as the lower part of the fork can vary along their length.

\subsection{Digitization and FE Models}

First, using a 3D Laser Scanner in order to collect, process and design the CAD model. Next, the geometry of the bicycle frame is discretized mainly by shell elements (triangular) and solid elements (tetrahedral). The total number of DOFs was 1,000,000. Similarly, the upper part of the fork was modeled with shell (triangular and quadrilateral) and solid (tetrahedral) elements resulting to $125,000 \mathrm{DOFs}$ and the lower part of the fork was modeled only with solid (tetrahedral) elements resulting to 205,000 DOFs. The detailed FE models are presented in Figures 2, 3 and 4. For the development and solution of the finite element models appropriate software was used $[25,26]$.

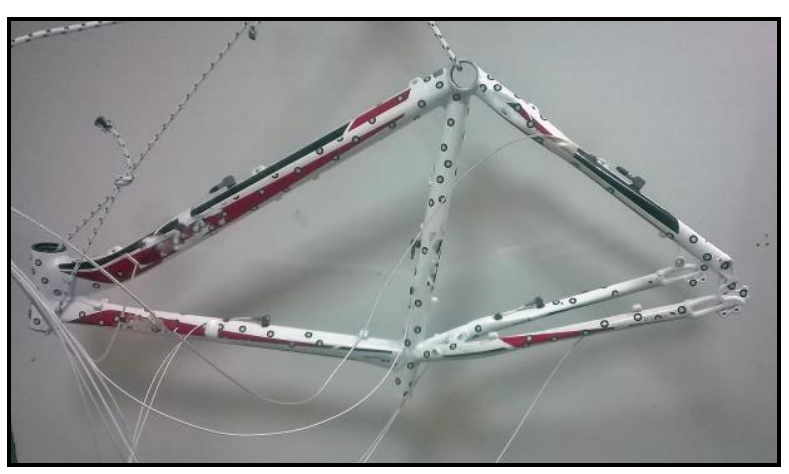

Figure 1: Bicycle frame
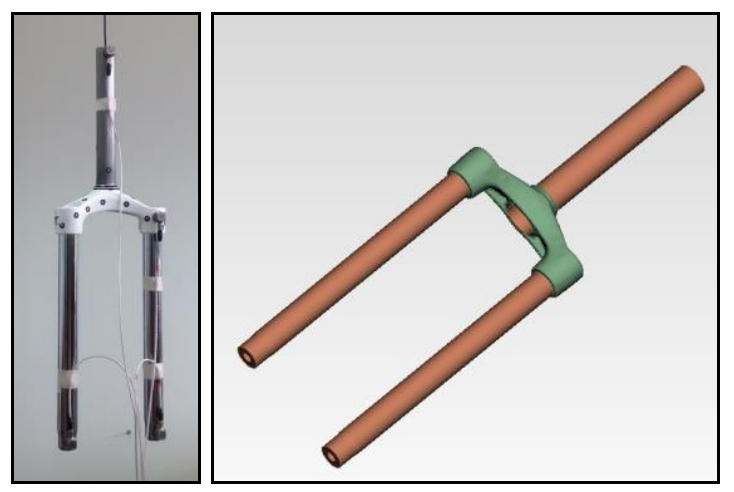

Figure 3: Upper (part 1) of the suspension-fork

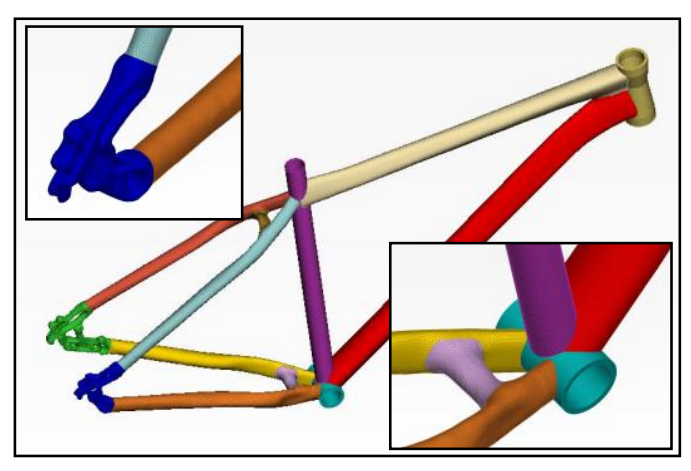

Figure 2: Finite element model of the frame

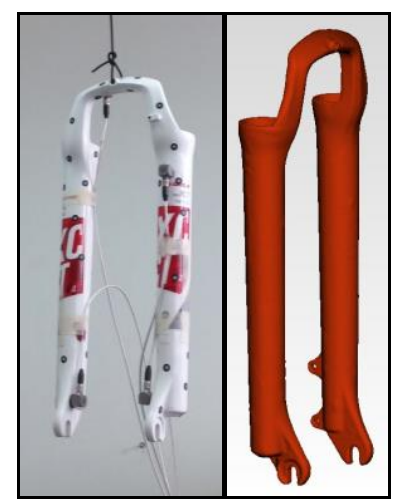

Figure 4: Lower (part 2) of the suspension-fork 


\subsection{Experimental Modal Analysis}

After developing the nominal finite element models, an experimental modal analysis of the frame and fork parts, was performed to quantify its dynamic characteristics. The frame and fork parts were hung up using a crane and straps, to approximate free-free boundary conditions for the test. First, all the necessary elements of the FRF matrix required for determining the response of the substructures were determined by imposing impulsive loading [5-9]. The measured frequency range was $0-2048 \mathrm{~Hz}$, which includes the analytical frequency range of interest for the frame, $0-600 \mathrm{~Hz}$, as well as for the fork parts, 0-500 Hz. An initial investigation indicated eleven natural frequencies for the frame and four for the fork parts, in this frequency range. A schematic illustration of the experimental arrangement is presented in Figure 5. In these figures, the locations and directions of acceleration measurements are presented, applying an impulsive load in all directions and at several locations. Based on the measured FR functions, the natural frequencies and the damping ratios of the substructures were estimated $[10,11,15]$. The identified mode shapes have also been recorded so that they can be used for updating the finite element models.
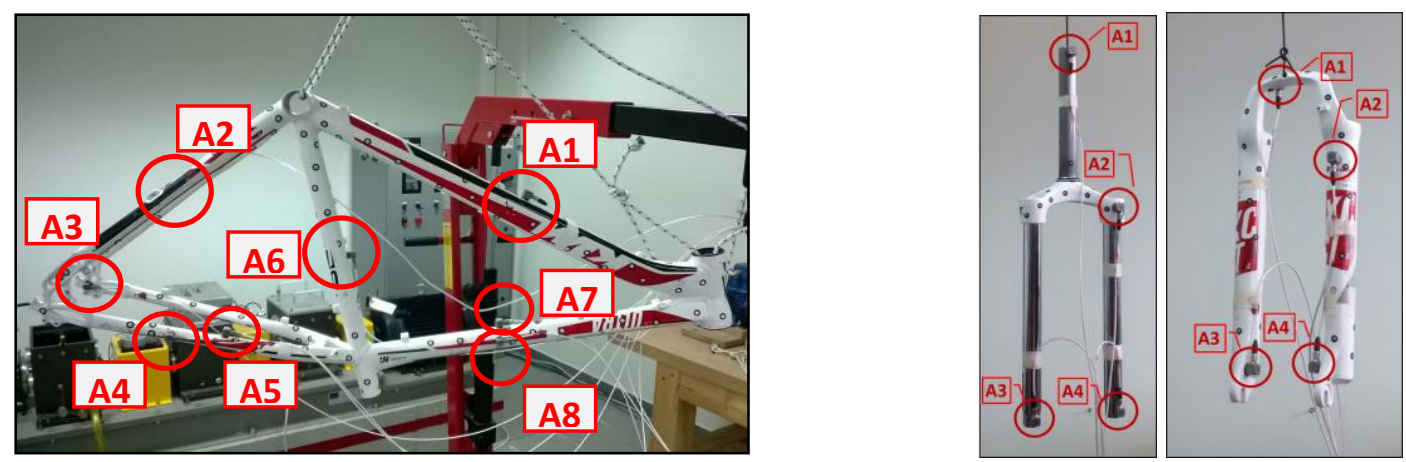

Figure 5: Accelerometer locations on main frame and fork parts 1 and 2

As an outcome of the above two procedures, in the Table 1 summarized the modal analysis results for the main frame, while similar results presented for the fork parts 1 and 2 in the Table 2.

\begin{tabular}{ccccc}
\hline Mode & $\begin{array}{c}\text { Identified } \\
\text { Modal } \\
\text { Frequency }\end{array}$ & $\begin{array}{c}\text { Nominal FE } \\
\text { Predicted } \\
\text { Modal } \\
\text { Frequency }\end{array}$ & $\begin{array}{c}\text { Difference } \\
\text { between } \\
\text { Identified and FE } \\
\text { Predicted Modal } \\
\text { Frequencies }\end{array}$ & $\begin{array}{c}\text { Identified } \\
\text { Modal } \\
\text { Damping } \\
\text { Ratio }\end{array}$ \\
\hline & $\omega_{r E}[H z]$ & $\omega_{r N_{F E}}[H z]$ & $\frac{\omega_{r N_{F E}}-\omega_{r E}}{\omega_{r N_{F E}}} 100 \%$ & $\zeta_{r E}(\%)$ \\
\hline $\mathbf{1}$ & 84.88 & 96.46 & 12.01 & 0.21 \\
$\mathbf{2}$ & 108.29 & 118.72 & 8.78 & 0.19 \\
$\mathbf{3}$ & 270.69 & 319.15 & 15.19 & 0.12 \\
$\mathbf{4}$ & 311.96 & 357.30 & 12.69 & 0.08 \\
$\mathbf{5}$ & 315.15 & 358.72 & 12.15 & 0.13 \\
$\mathbf{6}$ & 368.01 & 414.69 & 11.26 & 0.13 \\
$\mathbf{7}$ & 425.73 & 491.11 & 13.31 & 0.12 \\
$\mathbf{8}$ & 450.25 & 494.05 & 8.87 & 0.16 \\
$\mathbf{9}$ & 465.19 & 530.79 & 12.36 & 0.16 \\
$\mathbf{1 0}$ & 484.45 & 545.44 & 11.18 & 0.35 \\
$\mathbf{1 1}$ & 504.86 & 575.88 & 12.33 & 0.23 \\
\hline
\end{tabular}

Table 1: Modal frequencies and modal damping ratios of the frame

\begin{tabular}{|c|c|c|c|c|}
\hline Mode & $\begin{array}{l}\text { Identified } \\
\text { Modal } \\
\text { Frequency }\end{array}$ & $\begin{array}{l}\text { Nominal FE } \\
\text { Predicted } \\
\text { Modal } \\
\text { Frequency }\end{array}$ & $\begin{array}{c}\text { Difference } \\
\text { between } \\
\text { Identified and FE } \\
\text { Predicted Modal } \\
\text { Frequencies }\end{array}$ & $\begin{array}{l}\text { Identified } \\
\text { Modal } \\
\text { Damping } \\
\text { Ratio }\end{array}$ \\
\hline & $\omega_{r E}[\mathrm{~Hz}]$ & $\omega_{r N_{F E}}[H z]$ & $\frac{\omega_{r N_{F E}}-\omega_{r E}}{\omega_{r N_{F E}}} 100 \%$ & $\zeta_{r E}(\%)$ \\
\hline \multicolumn{5}{|c|}{ FORK PART 1} \\
\hline 1 & 188.26 & 168.18 & 11.94 & 0.15 \\
\hline 2 & 268.79 & 259.38 & 3.63 & 0.11 \\
\hline 3 & 419.36 & 453.39 & 7.51 & 0.39 \\
\hline 4 & 452.13 & 499.42 & 9.47 & 0.43 \\
\hline \multicolumn{5}{|c|}{ FORK PART 2} \\
\hline 1 & 79.65 & 80.88 & 1.51 & 0.48 \\
\hline 2 & 86.21 & 94.45 & 8.72 & 0.15 \\
\hline 3 & 425.04 & 429.20 & 0.97 & 0.12 \\
\hline 4 & 497.25 & 553.03 & 10.09 & 0.11 \\
\hline
\end{tabular}

Table 2: Modal frequencies and modal damping ratios of the two parts of the suspension-fork 
More specifically, the first column of Table 1 presents the values of the lowest natural frequencies $\left(\omega_{r E}\right)$, while the corresponding damping ratios are included in the fourth column. In the same table, the second column presents the values of the natural frequencies obtained from the analysis of the nominal finite element model $\left(\omega_{r N_{F E}}\right)$ and the third column compares these frequencies with the corresponding frequencies obtained by the experimental data. The errors determined between the nominal FE model and the experimental measurements are not insignificant, indicating that the FE model updating process is necessary.

\section{FINITE ELEMENT MODEL UPDATING}

\subsection{Linear FE model parameterization}

The parameterization of the finite element models is introduced in order to demonstrate the applicability of the proposed finite element model updating method. The parameterized models are consisted of thirteen, two and four parts, for the frame, fork part 1 and fork part 2, respectively, as shown in Figure 6. The first four parts (P1, P2, P3 and P4) of the frame are modeled with solid elements, while the remaining nine parts (P5-P13) are modeled with shell elements. Fork part 1 is modeled with shell and solid elements for P1 and P2 respectively and fork part 2 is modeled solely with solid elements. The thickness of the shell elements, the Young's modulus and the density are used as design variables. Thus, the final numbers of the design parameters are thirty-five (35), five (5) and eight (8) variables for the frame, fork part 1 and part 2 respectively.
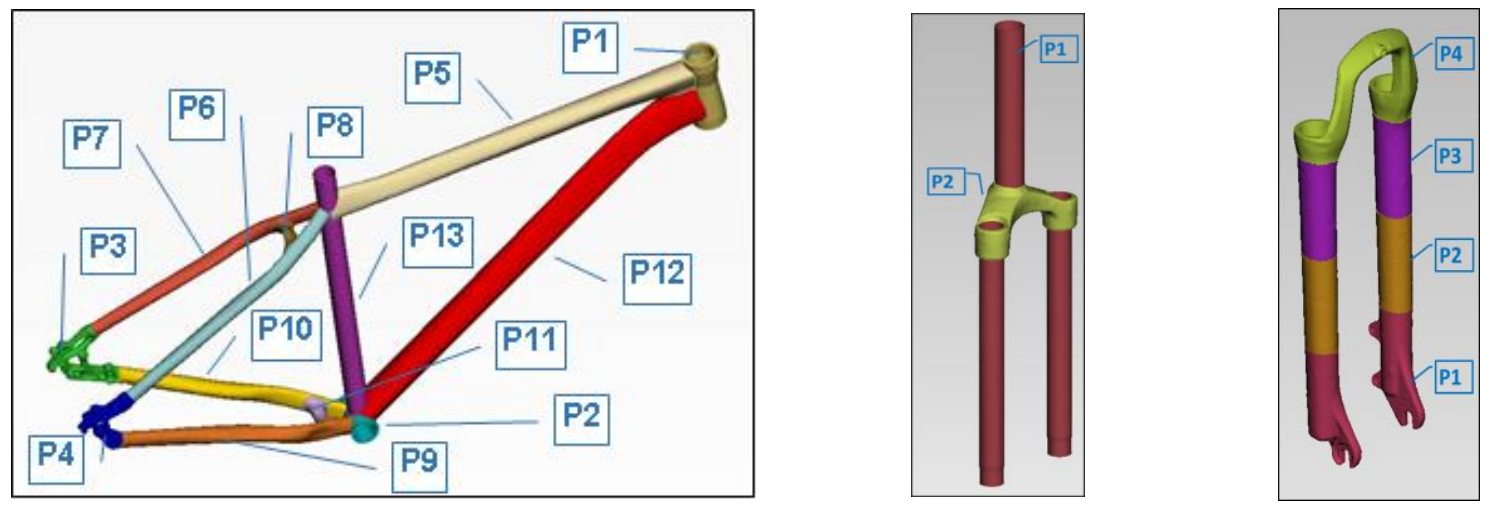

Figure 6: Parts of the parameterized FE Models of the frame and suspension- fork parts

The FE models of the main frame and the fork parts are updated using the identified modal frequencies and mode shapes shown in Tables 1 and 2. The identified mode shapes include components at all sensor locations. Additionally, we define as design response the total weight of the model, in order to be taken into consideration during the optimization process.

\subsection{Linear FE Model Updating Results}

The results from the FE model updating method are shown in Tables 3 and 4 for the frame and fork parts respectively. A comparison between identified $\left(\omega_{r E}\right)$ and optimal FE predicted modal frequencies $\left(\omega_{r O_{F E}}\right)$ is also presented.

The FRF predicted by the optimal FE model (black dashed line) for the frame are compared in Figure 7 with the FRF computed directly from the measured data (red continuous line) at two indicative measurement locations in the frequency range $[50 \mathrm{~Hz}$, $550 \mathrm{~Hz}]$. The FRF of the initial nominal model (blue dashed dot line) is also shown in these 
figures to be inadequate to represent the measured FRF. Compared to the FRF of the initial nominal model, it is observed that the updated optimal model tend to considerably improve the fit between the model predicted and the experimentally obtained FRF close to the resonance peaks.

\begin{tabular}{cccc}
\hline Mode & $\begin{array}{c}\text { Identified } \\
\text { Modal } \\
\text { Frequency }\end{array}$ & $\begin{array}{c}\text { Optimal FE } \\
\text { Predicted } \\
\text { Modal } \\
\text { Frequency }\end{array}$ & $\begin{array}{c}\text { Difference } \\
\text { between Identified } \\
\text { and FE Predicted } \\
\text { Modal Frequencies }\end{array}$ \\
\hline & $\omega_{r E}[H z]$ & $\omega_{r O_{F E}}[H z]$ & $\frac{\omega_{r O_{F E}}-\omega_{r E}}{\omega_{r O_{F E}}} 100 \%$ \\
\hline $\mathbf{1}$ & 84.88 & 84.89 & 0.01 \\
$\mathbf{2}$ & 108.29 & 108.29 & 0.00 \\
$\mathbf{3}$ & 270.69 & 270.61 & 0.03 \\
$\mathbf{4}$ & 311.96 & 312.16 & 0.06 \\
$\mathbf{5}$ & 315.15 & 315.47 & 0.10 \\
$\mathbf{6}$ & 368.01 & 368.01 & 0.00 \\
$\mathbf{7}$ & 425.73 & 426.01 & 0.07 \\
$\mathbf{8}$ & 450.25 & 449.51 & 0.17 \\
$\mathbf{9}$ & 465.19 & 465.59 & 0.09 \\
$\mathbf{1 0}$ & 484.45 & 484.38 & 0.02 \\
$\mathbf{1 1}$ & 504.86 & 505.23 & 0.07 \\
\hline
\end{tabular}

Table 3: Comparison between identified and optimal FE predicted modal frequencies for the main frame.

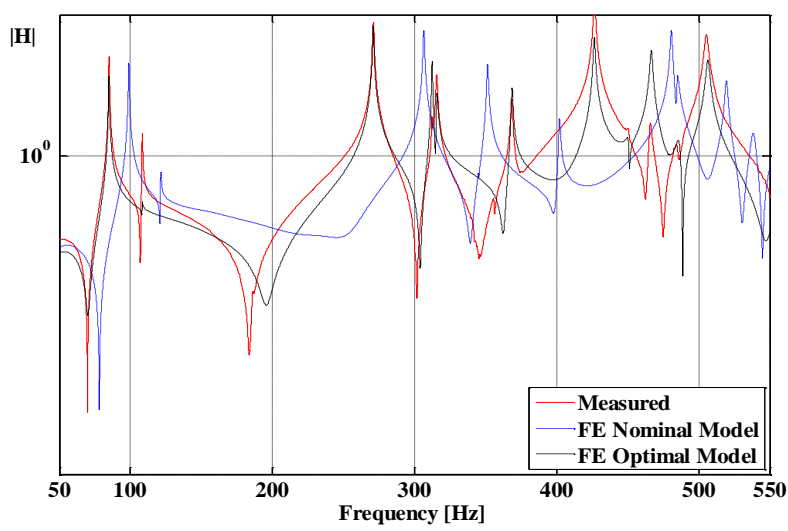

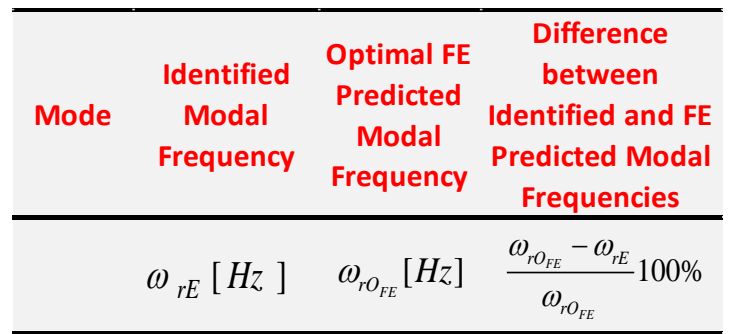

\begin{tabular}{lccc}
\hline \multicolumn{4}{c}{ FORK PART 1 } \\
\hline $\mathbf{1}$ & 188.26 & 188.53 & 0.14 \\
$\mathbf{2}$ & 268.79 & 267.86 & 0.35 \\
$\mathbf{3}$ & 419.36 & 420.83 & 0.35 \\
$\mathbf{4}$ & 452.13 & 452.10 & 0.01 \\
\hline \multicolumn{4}{c}{ FORK PART 2 } \\
\hline $\mathbf{1}$ & 79.65 & 79.65 & 0.00 \\
$\mathbf{2}$ & 86.21 & 86.21 & 0.00 \\
$\mathbf{3}$ & 425.04 & 425.04 & 0.00 \\
$\mathbf{4}$ & 497.25 & 497.25 & 0.00 \\
\hline
\end{tabular}

Table 4: Comparison between identified and optimal FE predicted modal frequencies for the fork parts $1 \& 2$.

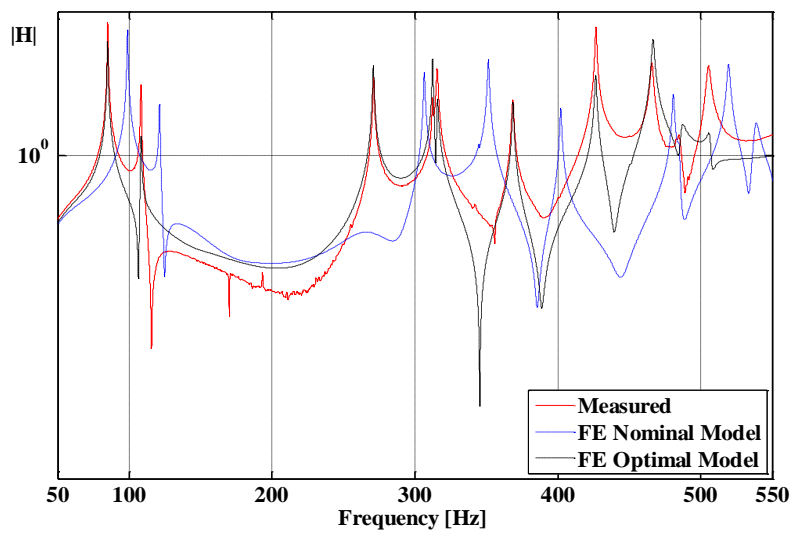

Figure 7: Comparison between measured, nominal and optimal FRF in two typical elements of the FRF matrix

\section{ANALYSIS OF NON-LINEAR SYSTEM}

The FE model, of the nonlinear subassembly of the suspension-fork, was created connecting fork part 1 and 2 with two springs simulating the restoring force $f_{r}(x)$ and two non-linear bushing elements simulating the damping force $f_{d}(\dot{x})$. The final nonlinear suspension FE model with the corresponding lumped model presented in Figure 8.

In order to identify the parameters of the subsystem, which exhibit strongly nonlinear characteristics, a series of experimental trials was performed. In the experimental device 
shown in Figure 9 two electromagnetic devices are used, at the top and at the bottom of the suspension, imposing harmonic excitation with $\pi / 2$ rad phase difference using $0.2 \mathrm{~Hz}$ frequency step for three different forcing levels.

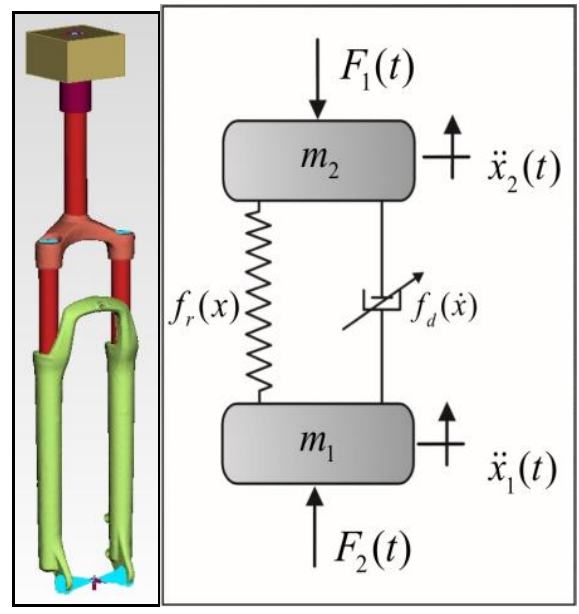

Figure 8: FE model of the suspension-fork and theoretical lumped model

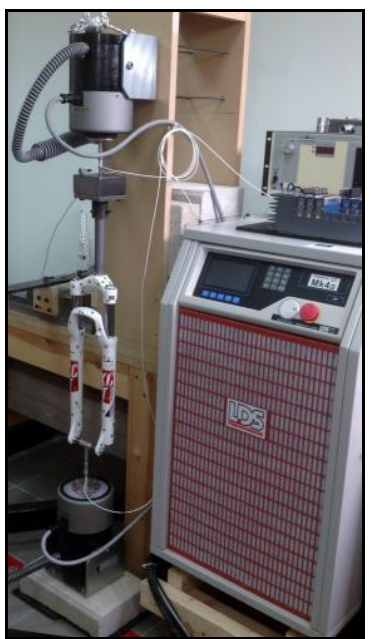

Figure 9: Schematic illustration and experimental arrangement of suspension sub-assembly

In Figure 10 depicted the imposed forces $F_{1}(t)$ and $F_{2}(t)$ for the three different forcing levels at $\omega=6 \mathrm{~Hz}$, which is approximately the resonant frequency of the system, while in Figure 11 depicted the acceleration transmissibility functions of measurement location 2, for the three different forcing levels. A number of models of the restoring and damping forces, say $f_{r}$ and $f_{d}$, respectively, were tried for modeling the action of the supports and compared with the experimental results. The classic linear dependence of the restoring force on the displacement and of the damping forces on the velocity of the support unit was first assumed. However, critical comparison with the experimental results using the Bayesian model selection framework demonstrated that the outcome was unacceptable in terms of accuracy. Eventually it was found that an acceptable form of the restoring forces is the one where they remain virtually in a linear relation with the extension of the spring, namely

$$
f_{r}(x)=k x
$$

while the damping force was best approximated by the following formula

$$
f_{d}(\dot{x})=c_{1} \dot{x}+\frac{c_{2} \dot{x}}{c_{3}+|\dot{x}|}
$$

As usual, the linear term in the last expression is related to internal friction at the support, while the nonlinear part is related to the existence and activation of dry friction. More specifically, in the limit $c_{3} \rightarrow 0$, the second term in the right hand side of Equation (8) represents energy dissipation action corresponding to dry friction. On the other side, in the limit $c_{3} \rightarrow \infty$ this term represents classical viscous action and can actually be absorbed in the first term.

The results for $k, c_{1}, c_{2}$ and $c_{3}$ are obtained based on the experimental response spectra values for the accelerations of the two suspension components, using the parameters of the updated linear parts (1 and 2) of the suspension fork subassembly and the transmissibility functions of the support system for three different forcing levels. Let $S=\left\{\hat{s}_{r}\left(\omega_{r}\right) \in R^{N_{r}}, r=1, \ldots, n\right\}$ 
be the measured transmissibility functions at $N_{r}$ measured frequencies where $n$ is the number of involved frequencies. Let $\underline{\theta}=\left(k_{1}, c_{1}, c_{2}, c_{3} \in R^{N_{\theta}}\right)$ be a parameter space, corresponding to the linear and nonlinear coefficients of the restoring and damping forces respectively.

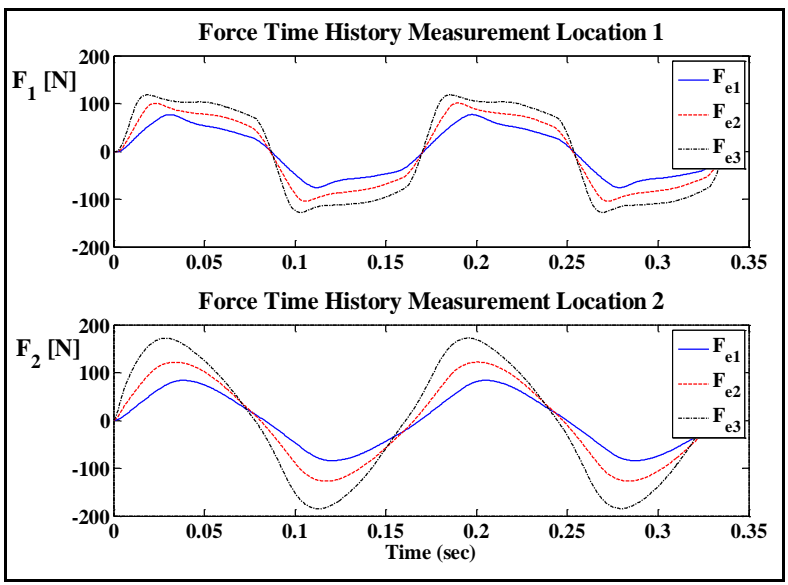

Figure 10: Time-history of the external forces applied at a fundamental harmonic frequency $\omega=6 \mathrm{~Hz}$

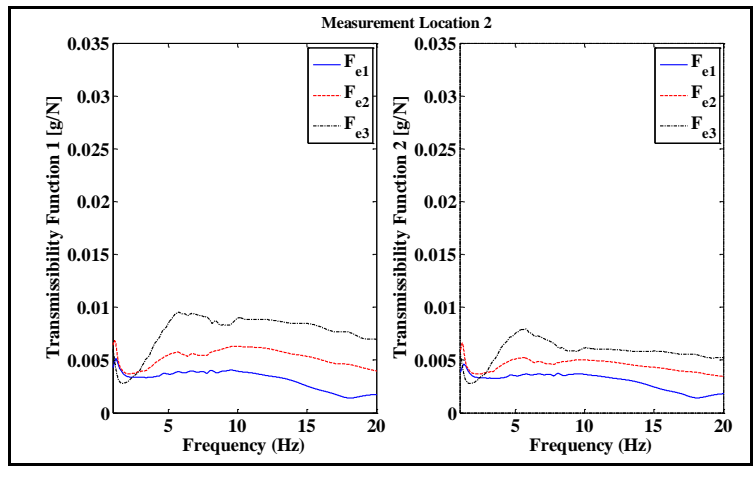

Figure 11: Transmissibility functions of the support system for three different forcing levels

The results for $k, c_{1}, c_{2}$ and $c_{3}$ are obtained based on the experimental response spectra values for the accelerations of the two suspension components, using the parameters of the updated linear parts (1 and 2) of the suspension fork subassembly and the transmissibility functions of the support system for three different forcing levels. Let $S=\left\{\hat{s}_{r}\left(\omega_{r}\right) \in R^{N_{r}}, r=1, \ldots, n\right\}$ be the measured transmissibility functions at $N_{r}$ measured frequencies where $n$ is the number of involved frequencies. Let $\underline{\theta}=\left(k_{1}, c_{1}, c_{2}, c_{3} \in R^{N_{\theta}}\right)$ be a parameter space, corresponding to the linear and nonlinear coefficients of the restoring and damping forces respectively. Similarly to the linear model, the objective is to estimate the values of the parameter set $\underline{\theta}$ so that the analytical response spectra values, namely the transmissibility functions match the experimentally obtained functions estimated in $S$. In Figure 12, presented a comparison in two measured locations, between the numerical nonlinear FE model (red line), with the experimental measurements (black line), in harmonic excitation with forcing frequency of $\omega=6 \mathrm{~Hz}$. More specifically, the nonlinear FE model with the estimated values of the stiffness and damping properties, was solved with nonlinear transient response analysis. The blue line was derived using the equivalent theoretical linear model and is presented also for comparison purposes.

From these results, it arises that the estimation of the values of the parameters is very close to the real values, since the theoretical nonlinear model is very close to those of the experimental results.

Finally, in order to test the reliability of the applied methodology, we examine the full bicycle model (Fork and frame). More specifically, with the use of two electrodynamic shakers imposing base excitation at the wheels locations and measuring the dynamic response in several locations in the frame and suspension substructures in the three directions. 
An illustration of the fixed-free arrangement of the bike on the shakers with acceleration measurement locations is presented in Figure 14, while in Figure 13 the finite element model of the whole bike assembly is shown.

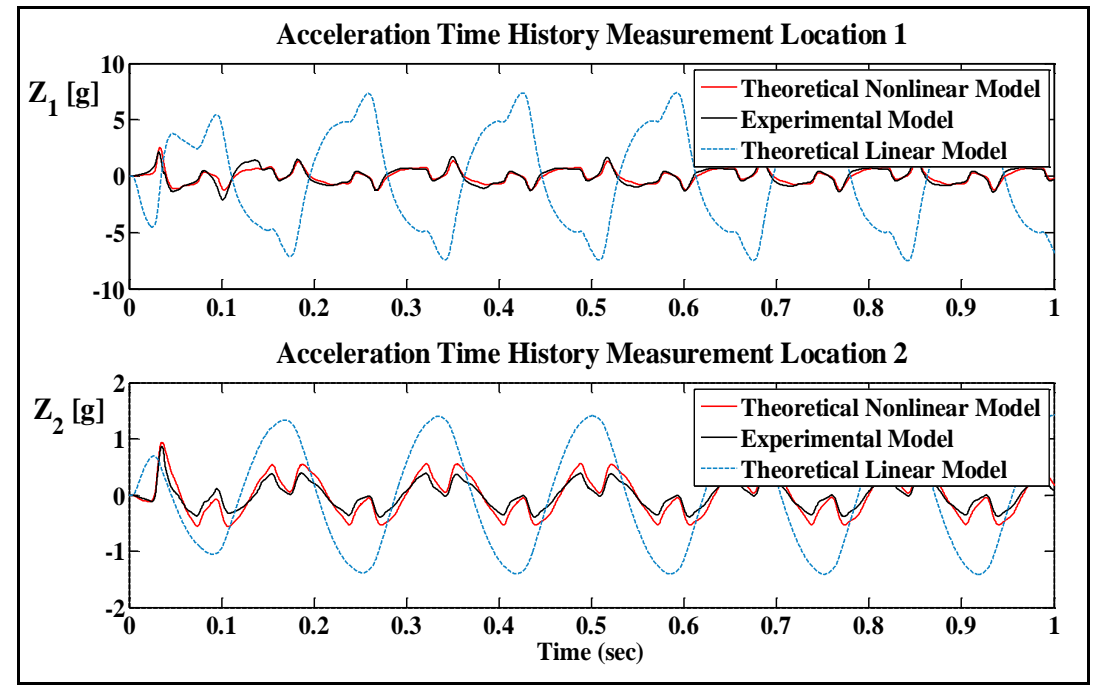

Figure 12: Acceleration time histories at two measured locations in harmonic excitation with frequency $\omega=6 H z$

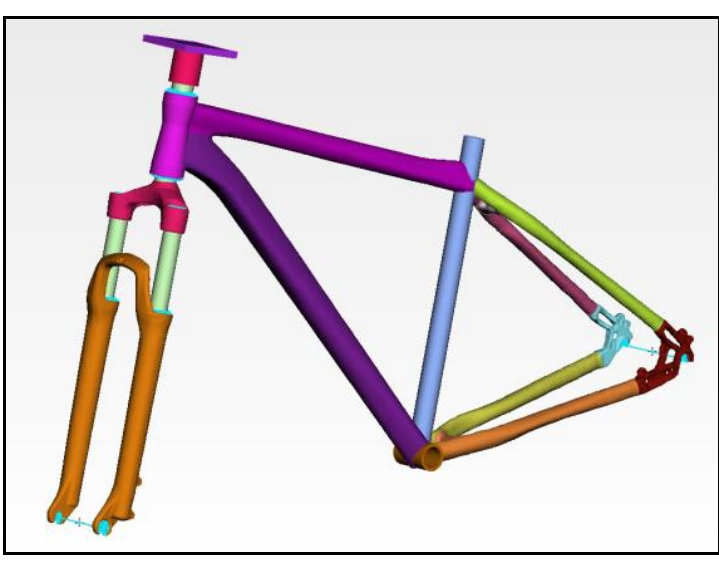

Figure 13: FE model of the bike assembly.

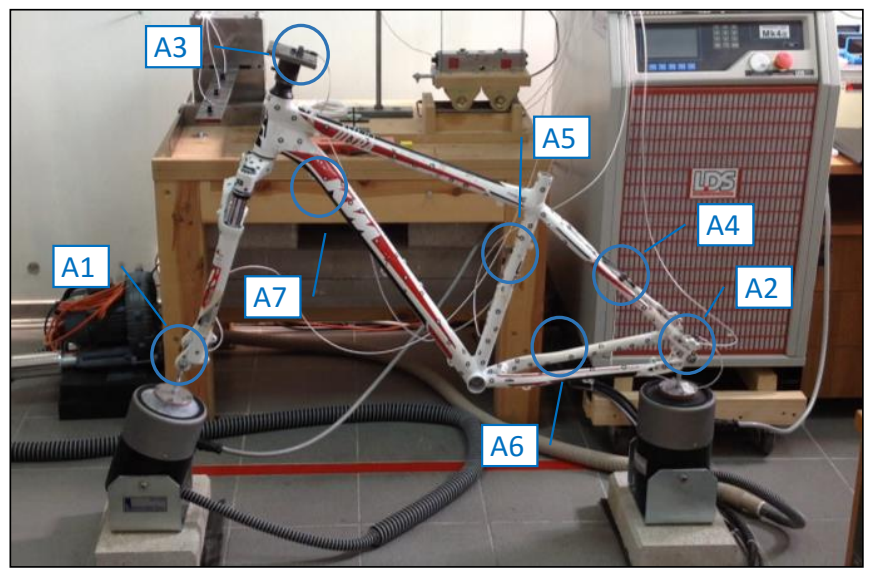

Figure 14: Fixed-free arrangement on electrodynamic shakers

The measured acceleration time histories in the three directions in the locations 1 and 2, were imported as base excitation in the finite element model of the system. In Figure 15, presented a comparison in three measured locations in the vertical direction, between the numerical nonlinear FE model (red line), with the experimental measurements (black line), in harmonic excitation with forcing frequency of $\omega=6 \mathrm{~Hz}$. The blue line was derived using the equivalent theoretical linear model and is presented again for comparison purposes.

From these results, it arises that the full bicycle theoretical nonlinear FE model is very close to those of the experimental results.

Also, in Figure 16, is shown an indicative point of time, of the dynamic response of the full nonlinear system. 

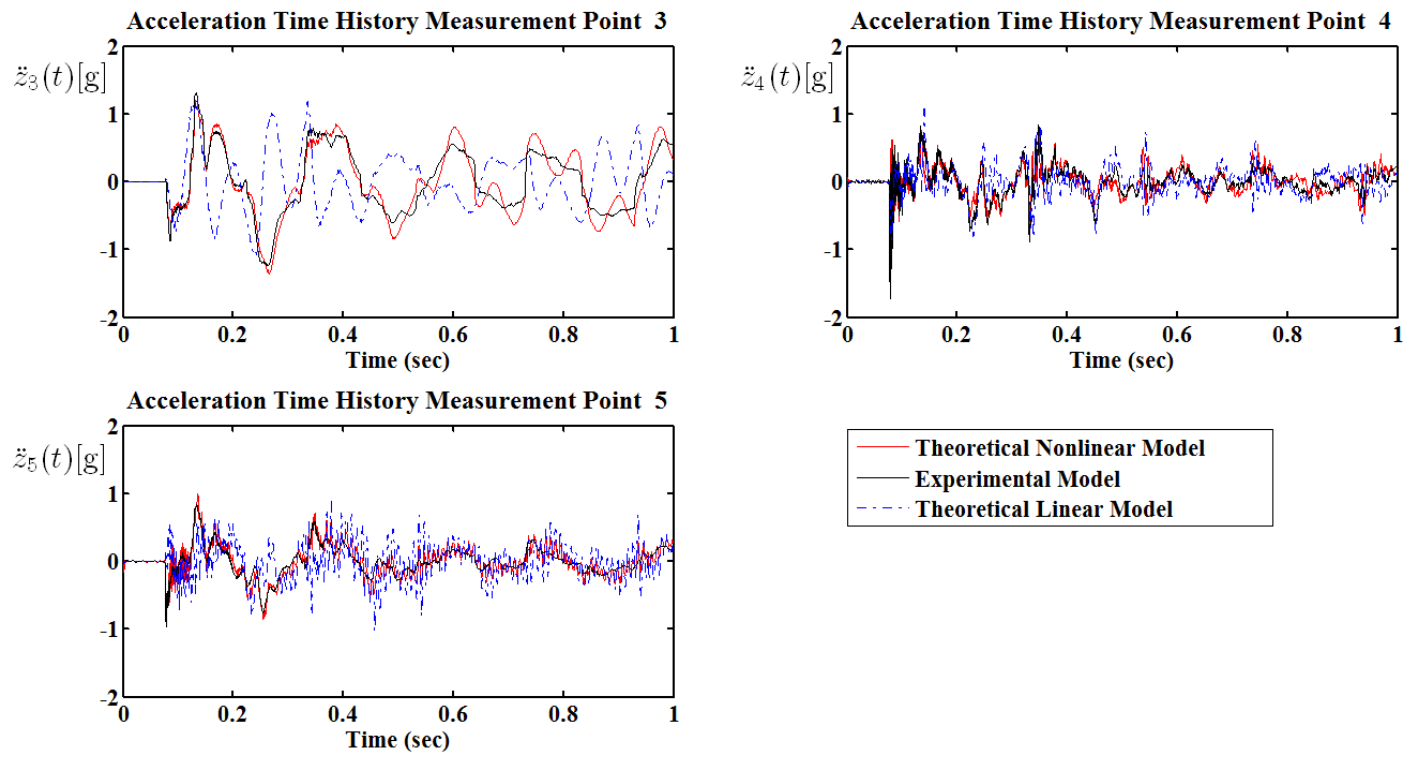

Figure 15: Acceleration time histories at three measured locations in harmonic excitation with frequency $\omega=6 H z$

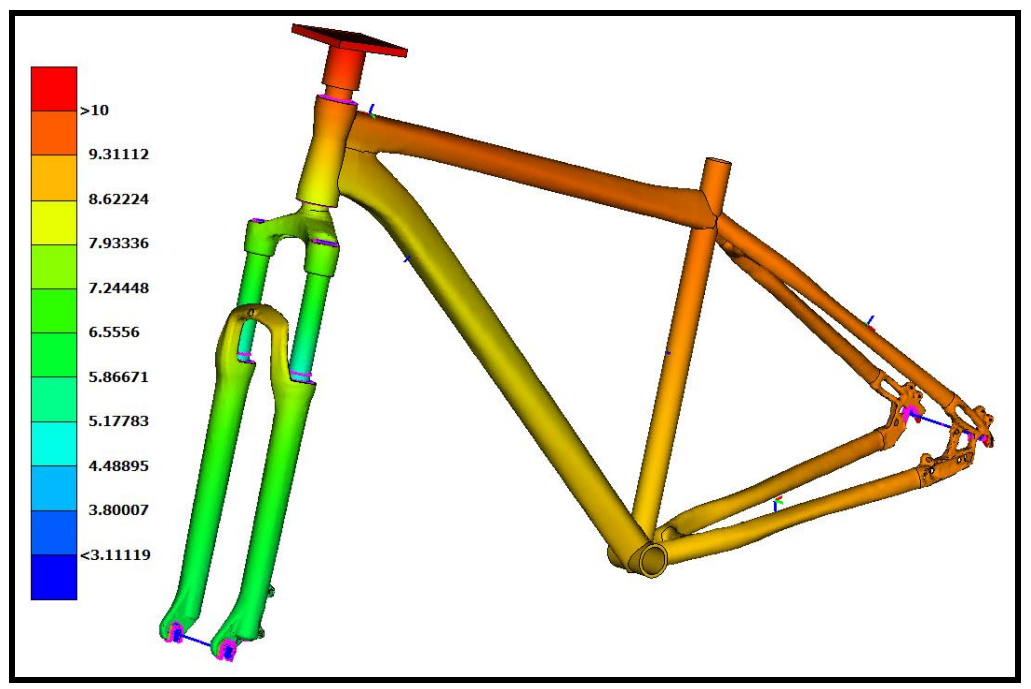

Figure 16: Dynamic response of the nonlinear full FE model in harmonic excitation with frequency $\omega=6 \mathrm{~Hz}$

\section{SUMMARY}

A computational framework is presented in order to estimate the optimal values of the linear and nonlinear components of large scale dynamical systems using vibration measurements. An extensible framework for Bayesian Uncertainty Quantification and Propagation of complex and computationally demanding physical models, was connected in an efficient way with a numerical code leading to an automated determination of dynamic response of linear and nonlinear mechanical systems. Numerical and experimental methodologies were applied in order to identify the model parameters and develop a high fidelity finite element model of the structure examined. The applicability and effectiveness of 
the methods applied, is explored by updating finite element model of a lightweight and geometrically complex bicycle assembly, using experimentally identified modal data. Direct comparison of the numerical and experimental data verified the reliability and accuracy of the methodology applied.

\section{REFERENCES}

[1] D. Rypl, Z. B. (2005). Generation of computational surface meshes of STL models. Prague, Czech Republic: Faculty of Civil Engineering, Department of Structural Mechanics, Czech Technical University.

[2] E. Bechét, J. C. (2002). Generation of a finite element MESH from stereolitography (STL) files. Paris, France: Computer-Aided Design

[3] Bianconi, F. (2002). Bridging the gap between CAD and CAE using STL files. Perugia, Italy: Department of Industrial Engineering, University of Perugia.

[4] Cuppens, K., Sas, P. and Hermans, L., Evaluation of FRF based substructuring and modal synthesis technique applied to vehicle FE data, ISMA 2000, K.U. Leuven, Belgium, pp. 1143-1150, 2000.

[5] Giagopoulos, D. and Natsiavas, S., Hybrid (numerical-experimental) modeling of complex structures with linear and nonlinear components, Nonlinear Dynamics, Vol. 47, pp. 193-217, 2007.

[6] Giagopoulos, D. and Natsiavas, S., Dynamic Response and Identification of Critical Points in the Superstructure of a Vehicle using a Combination of Numerical and Experimental Methods, Experimental Mechanics, DOI 10.1007/s11340-014-9966-z, 2014.

[7] Ewins, D.J., 1984, Modal Testing: Theory and Practice, Research Studies Press, Somerset, England.

[8] Mohanty, P. and Rixen, D.J., Identifying mode shapes and modal frequencies by operational modal analysis in the presence of harmonic excitation, Experimental Mechanics, Vol. 45, pp. 213-220, 2005.

[9] Spottswood, S.M. and Allemang, R.J., On the investigation of some parameter identification and experimental modal filtering issues for nonlinear reduced order models, Experimental Mechanics, Vol. 47, pp. 511-521, 2007.

[10]Richardson, M.H. and Formenti, D.L., Global curve fitting of frequency response measurements using the rational fraction polynomial method, Third IMAC Conference, Orlando, Florida, 1985.

[11] Mottershead, J.E. and Friswell, M.I., Model updating in structural dynamics: A survey, Journal of Sound and Vibration, Vol. 167, pp. 347-375, 1997.

[12]Papadimitriou, C., Ntotsios, E., Giagopoulos, D. and Natsiavas, S., Variability of updated finite element models and their predictions consistent with vibration measurements, Structural Control and Health Monitoring, Vol. 19, pp. 630-654, 2012. 
[13] Giagopoulos, D., Papadioti, D.-Ch., Papadimitriou, C. and Natsiavas, S., Bayesian uncertainty quantification and propagation in nonlinear structural dynamics, Proceedings of the IMAC-XXXI 2013, Garden Grove, California, USA, 2013.

[14]Christodoulou K, Ntotsios E, Papadimitriou C, Panetsos P. Structural model updating and prediction variability using Pareto optimal models. Computer Methods in Applied Mechanics and Engineering 2008; 198 (1):138-149.

[15]Ntotsios E, Papadimitriou C. Multi-objective optimization algorithms for finite element model updating. International Conference on Noise and Vibration Engineering (ISMA2008); Katholieke Universiteit Leuven, Leuven, Belgium, September 15-17, 2008.

[16] Mottershead J, Link M, Friswell M. The sensitivity method in finite element model updating: A tutorial. Mechanical Systems and Signal Processing. DOI 10.1016/j.ymssp.2010.10.012, 2010

[17]Grafe H Model Updating of Large Structural Dynamics Models Usong Measured Response Functions. Imperial College of Science, Department of Mechanical Engineering, Dynamics Section, Phd Thesis, 1998

[18] Fritzen CP, Jennewein D, Kiefer T. Damage detection based on model updating methods. Mechanical Systems and Signal Processing 1998; 12(1):163-186.

[19] Moaveni B, He X, Conte JP, Restrepo JI. Damage identification study of a seven-story full-scale building slice tested on the UCSD-NEES shake table. Structural Safety 2010; 32(5):347-356.

[20] Moaveni B, He X, Conte JP, De Callafon RA. Damage identification of a composite beam using finite element model updating. Computer-Aided Civil and Infrastructure Engineering 2008; 23(5):339-359.

[21] Weber B, Paultre P, Proulx J. Structural damage detection using nonlinear parameter identification with Tikhonov regularization. Structural Control and Health Monitoring 2007; 14:406-427.

[22] Yuen K-V, Beck JL, Katafygiotis LS. Efficient model updating and health monitoring methodology using incomplete modal data without mode matching. Structural Control and Health Monitoring 2006; 13:91-107.

[23] Allemang, R. and D. Brown (1982). A correlation coefficient for modal vector analysis. In 1st International Modal Analysis Conference, Orlando, USA.

[24] Grafe, H. (1995, May). Review of frequency response function updating methods. Technical report no. 1.01, BRITE-URANUS BRE2-CT94-0946.

[25]DYNAMIS 3.1.1, Solver Reference Guide, DTECH, Thessaloniki, Greece, 2013.

[26] ANSA and META-Post, BETA CAE Systems S.A., Thessaloniki, Greece. 